\title{
Pseudoaneurysm of a Bronchial Artery: An Unusual Cause of Chest Pain
}

\author{
Pepijn Van Hove ${ }^{1}$, Thomas Jardinet ${ }^{2}$ \\ ${ }^{1}$ Internal Medicine, University Hospital Antwerp, Antwerp, Belgium \\ ${ }^{2}$ Department of Interventional Radiology, University Hospital Antwerp, Antwerp, Belgium
}

Received: $16 / 01 / 2022$

Accepted: $19 / 01 / 2022$

Published: $17 / 02 / 2022$

How to cite this article: Van Hove P, Jardinet T. Pseudoaneurysm of a bronchial artery: an unusual case of chest pain. EJCRIM 2022;9: doi:10.12890/2022_003195.

Conflicts of Interests: The authors declare there are no competing interests.

This article is licensed under a Commons Attribution Non-Commercial 4.0 License

\section{ABSTRACT}

We report a case of a bronchial artery pseudoaneurysm presenting as acute retrosternal pain. We want to discuss and to announce the extremely rare finding of a bronchial artery pseudoaneurysm. Bronchial artery aneurysms and pseudoaneurysms are uncommon; however, missing this diagnosis is associated with significant morbidity and mortality. When suspecting this pathology urgent CT angiography and selective angiography (DSA) are crucial. Urgent treatment with transarterial embolization is preferred.

\section{LEARNING POINTS}

- Bronchial artery pseudoaneurysms are uncommon; however, missing this diagnosis is associated with significant morbidity and mortality.

- Haemoptysis and chest pain are the most common symptoms of bronchial artery pseudoaneurysms.

- Diagnosis is made with CT angiography and selective angiography (DSA). Transarterial embolization is the preferred type of treatment.

\section{KEYWORDS}

Bronchial artery pseudoaneurysm, mediastinal haematoma, digital subtraction angiography, embolization

\section{INTRODUCTION}

Aneurysms of the systemic bronchial arteries (BAA) are rare, with approximately 60 cases described in the literature ${ }^{[1]}$. Symptoms depend on the anatomic location, either mediastinal or intrapulmonary, and whether it has ruptured ${ }^{[2,3]}$. A similar but even more infrequent phenomenon is a pseudoaneurysm of a bronchial artery (BAP) ${ }^{[4]}$. Contrary to a true aneurysm in which all 3 layers of the arterial wall are involved, a false aneurysm (pseudoaneurysm) represents a contained rupture where not all layers are involved ${ }^{[2]}$. When a rupture occurs, symptoms can be retrosternal pain, epigastric pain, haemoptysis, dysphagia or hypovolaemic shock.

In this case report we present a case of a BAP with mediastinal bleeding and fulminant haemothorax.

\section{CASE DESCRIPTION}

An 85-year-old woman presented initially at the emergency department because of somnolence and loss of consciousness. Glasgow Coma Scale (GCS) was 10/15 (E3, V3, M4). She was febrile $\left(38.0^{\circ}\right)$ and oxygen saturation $\left(\mathrm{SpO}_{2}\right)$ was $97 \%$ on room air. Laboratory results showed

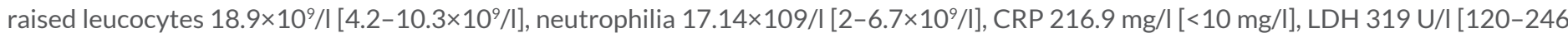
$\mathrm{U} / \mathrm{I}]$ and CK $239 \mathrm{U} / \mathrm{I}$ [34-145 U/I]. A CT scan of the chest and abdomen showed ground-glass opacities in both lobes of the left lung. No pulmonary embolism was seen. Two nasopharyngeal swabs were both negative for SARS-CoV-2 with real-time polymerase chain reaction (PCR) assessment. The patient was admitted to the acute geriatrics department. She received treatment with intravenous piperacillin- 
tazobactam over 3 days with downgrading to amoxicillin-clavulanic acid because of clinical and biochemical improvement. The patient recovered well with this therapy. The diagnosis of a community-acquired pneumonia with secondary loss of consciousness was made.

Four days after initial admission she complained of acute retrosternal pain, diaphoresis and shortness of breath. There was no haematemesis, haematochezia or haemoptysis. The patient was hyperventilating with mild tachycardia of 95 beats per minute and blood pressure of 176/86 mmHg. An electrocardiogram and troponins were both negative. The pain evolved to more epigastric pain. Because of high suspicion of a gastric ulcer, she was treated with intravenous pantoprazole and pain medication. Thirty-six hours later the patient complained of progressive pain and radiation to the interscapular space. She also complained of dysphagia. Because of the high suspicion of aortic dissection, CT angiography was performed. This demonstrated haemorrhagic fluid in the mediastinum and the right pleural space. Furthermore, a small enhancing mass in the infracarinal space was noticed, representing a pseudoaneurysm (Fig. 1). The latter originated from a bronchial artery arising from the inferior aspect of the aortic arch (Fig. 2).

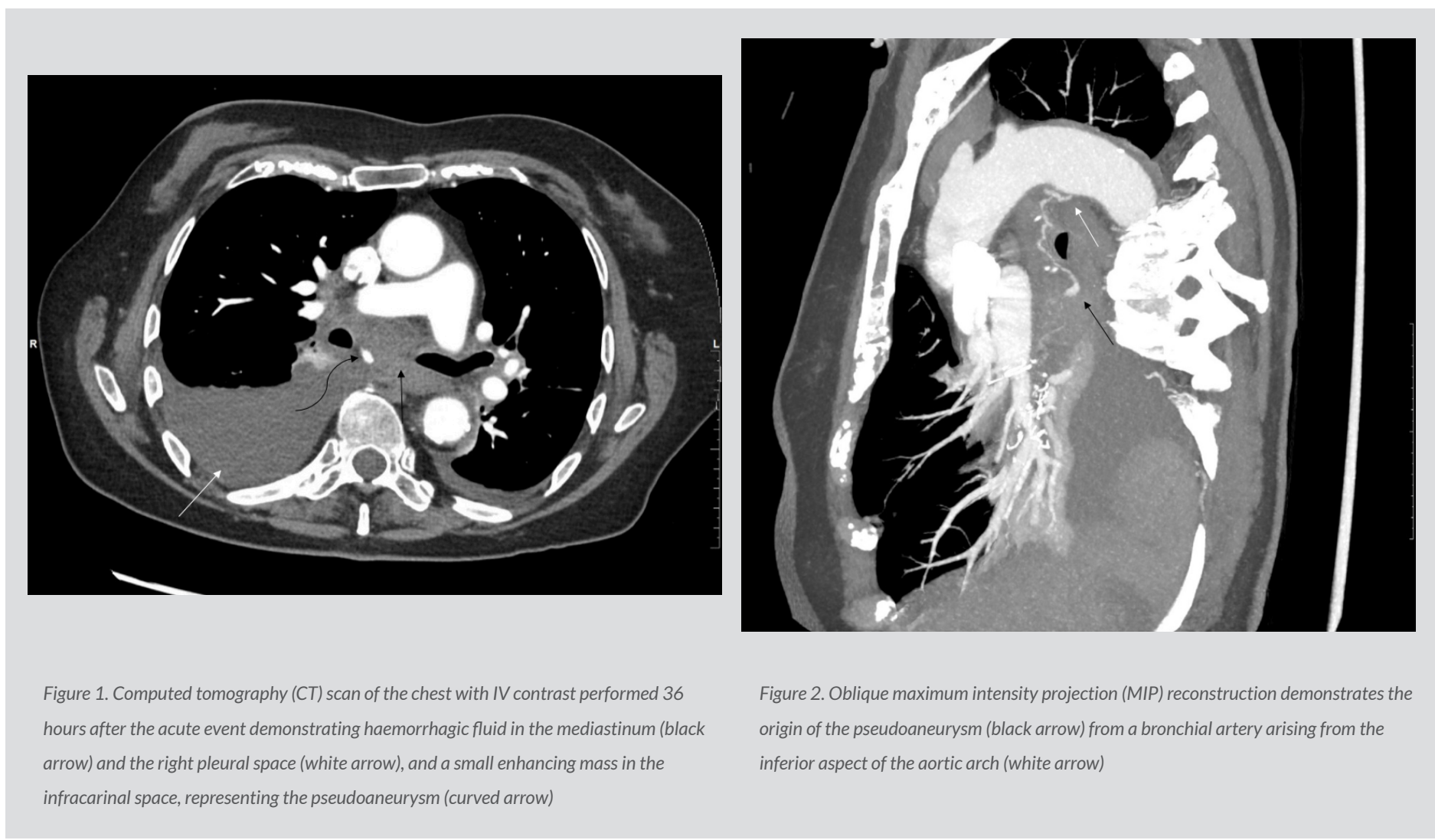

The patient was immediately taken to the interventional radiology department. Through the left radial arteryselective bronchial arteriography was performed (DSA). This confirmed the BAP (Fig. 3). More distal catheterization with a microcatheter (2.4F Direxion, Boston Scientific, Marlborough, USA) was followed by embolization with a 1:3 mixture of N-butyl cyanoacrylate (NBCA, Histoacryl, B. Braun, Melsungen, Germany) and ethiodized oil (Lipiodol, Guerbet, Villepinte, France). Complete cessation of flow in the target artery was achieved.

A CT scan performed 4 days before the acute event demonstrated the same bronchial artery with absence of any aneurysmal dilatation and any mediastinal haemorrhagic fluid (Fig. 4). This proves the diagnosis of an acute pseudoaneurysm in this bronchial artery. The patient could leave the hospital 27 days after the acute event.

\section{DISCUSSION}

The exact origin of a BAP is still not known because of its rarity and a lack of animal models. Probably it is secondary to an increased shear stress on a weakened arterial wall, as seen in bronchopulmonary infection, hypertension, tachycardia, obstructive lung diseases and trauma ${ }^{[1]}$. In this case, bronchopulmonary infection and elevated systemic systolic blood pressure may have contributed to the injury of the arterial wall and subsequent formation of the pseudoaneurysm. 


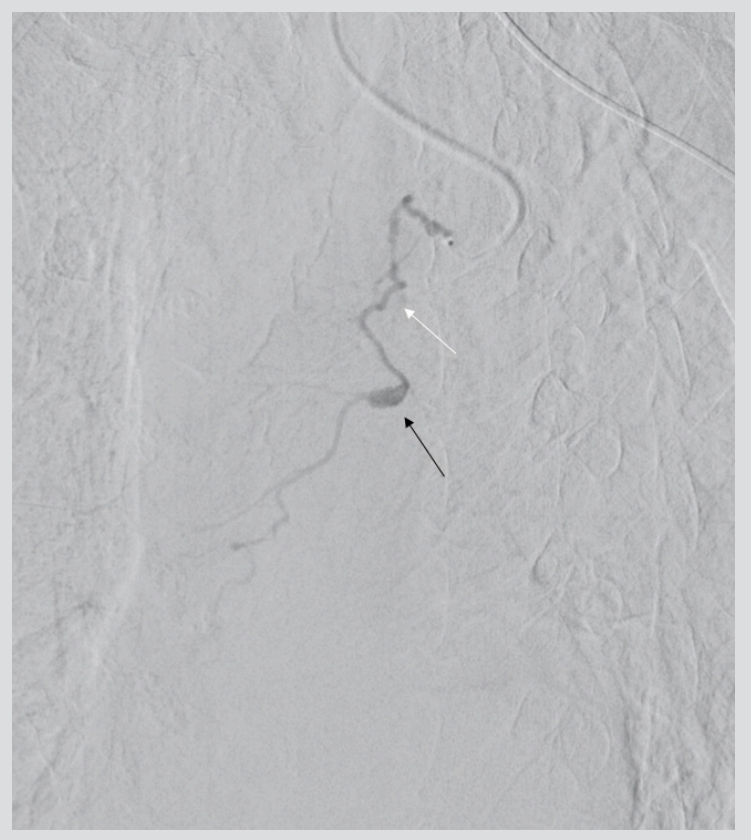

Figure 3. Selective angiography confirms the pseudoaneurysm (black arrow) arising from the bronchial artery (white arrow)

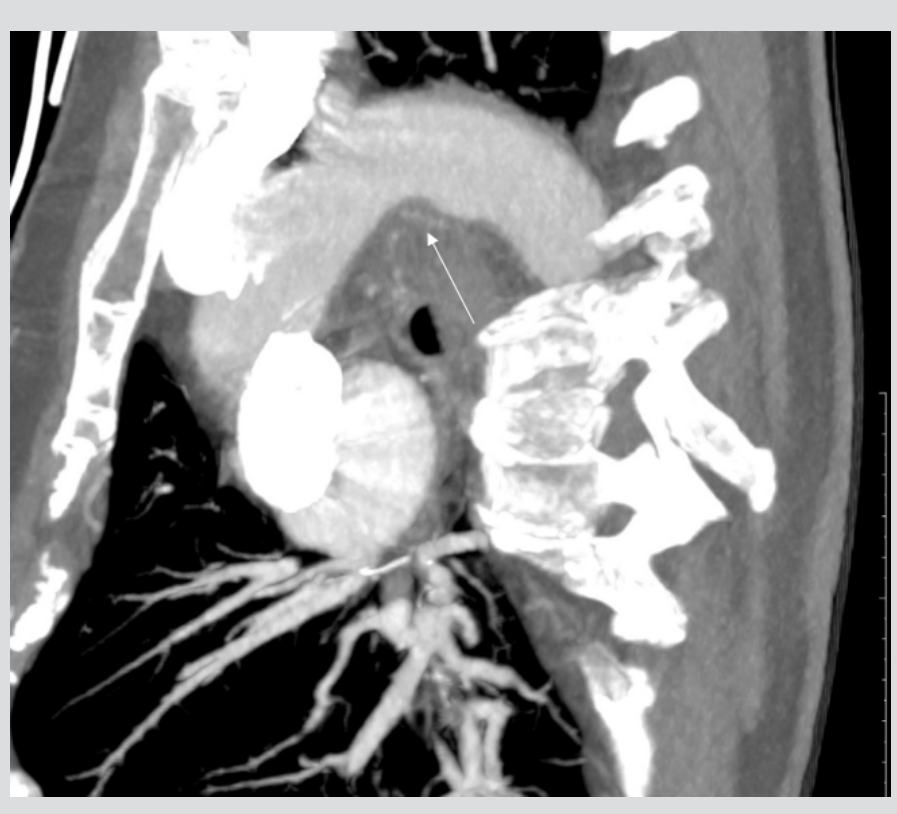

Figure 4. Similar MIP reconstruction of a CT scan performed 4 days before the acute event demonstrates the same bronchial artery (white arrow), with absence of any aneurysmal dilatation or any mediastinal haemorrhagic fluid

Many aneurysms remain asymptomatic while most of the pseudoaneurysms present with symptoms of the associated haematoma or haemorrhage ${ }^{[5]}$. Bronchial arteries are under systemic pressures so rupture could cause massive bleeding. A mediastinal haematoma could cause compressive symptoms, such as dysphagia or superior vena cava syndrome. Intrapulmonary pseudoaneurysms could present with symptoms of haemoptysis ${ }^{[6]}$. In this case, the patient complained initially of acute retrosternal pain, which after 36 hours evolved to epigastric and interscapular pain with associated dysphagia. This shift in symptoms should alert the clinician to developing mediastinal bleeding. Dysphagia developed due to the mass effect and the increasing pressure of the mediastinal haematoma compressing the oesophagus ${ }^{[5]}$. Acute retrosternal/epigastric pain in combination with new, progressive dysphagia should alert the clinician to carry out urgent CT angiography.

In this case, the acute event in combination with the absence of any aneurysmal dilatation of the bronchial artery on a CT scan 4 days earlier, suggests that the pseudoaneurysm formation was preceded by a dissection of this bronchial artery.

Once diagnosis is made with CT angiography and selective arteriography (DSA), (pseudo)aneurysm must be treated promptly, regardless of symptoms or size ${ }^{[1]}$.

In pulmonary and bronchial artery (pseudo)aneurysms, the endovascular approach (TAE) is the preferred treatment with fewer complications in comparison with surgery ${ }^{[1,7]}$. The aim of the embolization is to close the (pseudo)aneurysm as well as the afferent and efferent artery. Different kinds of embolic materials can be used to achieve this: gelatine sponge, coils, embolic particles and NBCA-Lipiodol mixture (glue) ${ }^{[1]}$. The most commonly used embolic agents are coils and glue ${ }^{[7]}$.

\section{CONCLUSION}

Bronchial artery aneurysms and pseudoaneurysms are uncommon; however, missing this diagnosis is associated with significant morbidity and mortality. When suspecting this pathology, urgent CT angiography and selective angiography (DSA) are crucial. Haemoptysis and chest pain are the most common symptoms. Transarterial embolization is the preferred type of treatment. This case is exceptional because of the radiological images performed 4 days before the acute event, which showed the absence of any aneurysmal pathology on the bronchial artery. With this case report, we hope to contribute to a better awareness of this rare clinical pathology. 


\section{REFERENCES}

1. Le-Jun F, Sun Y, Fan Y, Jin S. The effect of transcatheter bronchial artery embolization in five patients with bronchial artery aneurysm. Postepy Kardiol Interwencyjne 2020;16(3):330-335.

2. Restrepo CS, Carswell AP. Aneurysms and pseudoaneurysms of the pulmonary vasculature. Semin Ultrasound CT MR 2012;33(6):552-566.

3. Cearlock JR, Fontaine AB, Urbaneja A, Spigos DG. Endovascular treatment of a posttraumatic bronchial artery pseudoaneurysm. $J$ Vasc Interv Radiol 1995;6(3):495-496.

4. Koirala A, Thapa A, Mahat S, Sapkota S, Sosa O. A rare case of ruptured bronchial artery pseudoaneurysm and its nonsurgical management with interventional techniques. Cureus 2020;12(9):e10502.

5. Kaufman C, Kabutey NK, Sgroi M, Kim D. Bronchial artery pseudoaneurysm with symptomatic mediastinal hematoma. Clin Imaging 2014;38(4):536-539.

6. Kabilan K, Gulati M, Banday IA, Tyagi R, Bhalla AS, Naranje P, et al. Myriad faces of active tuberculosis: intrapulmonary bronchial artery pseudoaneurysm. Vasc Endovascular Surg 2022;56(2):212-215.

7. Kalra-Lall A, Donaldson J, Martin C 3rd. Brief review: pulmonary artery aneurysms and pseudoaneurysms. Int J Cardiovasc Imaging 2019;35(7):1357-1364. 